\title{
Estudo comparado da taxocenose de peixes em dois ambientes aquáticos do Parque Estadual de Itapuã, sul do Brasil
}

\author{
Ana Paula S. Dufech \& Clarice Bernhardt Fialho
}

Laboratório de Ictiologia, Departamento de Zoologia, Universidade Federal do Rio Grande do Sul, Av. Bento Gonçalves, 9500, 90540-000 Porto Alegre, RS, Brasil. (apdufech@hotmail.com)

\begin{abstract}
Comparative study of taxocenoses of fishes from two aquatic environments of the Parque Estadual de Itapuã, southern Brazil. This paper characterizes and compares the taxocenoses of fish from two different aquatic environments within a basin hidrographyc: the Praia das Pombas (Lago Guaíba) and the Lagoa Negra, both in the Parque Estadual de Itapuã, Rio Grande do Sul, considering quali-quantitative composition of the ichthyofauna. Ecological and abundance indexes were evaluated in both spatial and temporal contexts, checking that occurs relationship between the structure of taxocenose these sites with environmental variables. We sampled a total of 61 species, with the richness the same in both locations (44 species). Both environments showed peculiar characteristics that reflected on the fish fauna in each location. It was observed that the pattern of distribution of the taxocenoses of fishes studied in the two environments is seasonal, since the abundance, biomass and diversity were higher in the hottest months of the year, spring and summer. The temperature and day-length the main factors that influenced in the distribution of the fish fauna of the Praia das Pombas and Lagoa Negra.
\end{abstract}

KEYWORDS. Praia das Pombas, Lagoa Negra, ichthyofauna, diversity, seasonality.

RESUMO. Este trabalho tem por objetivos caracterizar e comparar a taxocenose de peixes de dois ambientes aquáticos distintos dentro de uma mesma bacia hidrográfica: a praia das Pombas (lago Guaíba) e a lagoa Negra, ambos no Parque Estadual de Itapuã, Rio Grande do Sul. Foram analisados aspectos que incluem a composição quali-quantitativa da ictiofauna. Para tanto, foram utilizados índices ecológicos e de abundância, sendo avaliados espacial e temporalmente e verificando se ocorre relação entre a estrutura da taxocenose destes locais e as variáveis ambientais. Foram amostradas 61 espécies no total, sendo a riqueza a mesma nos dois ambientes (44 espécies). Os dois ambientes estudados apresentam características peculiares, refletindo na composição de espécies de cada um destes locais. Foi possível observar que o padrão de distribuição da taxocenose de peixes estudada nos dois ambientes é sazonal, uma vez que a abundância, a biomassa e a diversidade foram mais elevadas nos meses mais quentes do ano, ou seja, primavera e verão. A temperatura e o fotoperíodo foram os principais fatores que influenciaram na distribuição da fauna de peixes da praia das Pombas e na lagoa Negra.

PALAVRAS-CHAVE. Praia das Pombas, lagoa Negra, ictiofauna, diversidade, sazonalidade.

Comunidades biológicas variam no tempo e no espaço, como resultado de diferenças na estrutura do hábitat (GORMAN \& KARR, 1978), disponibilidade de recursos (Grenoulllet et al., 2002) e padrões biogeográficos (JACKSON \& HARVEY, 1989; TONN et al., 1990; MATTHEWs \& RoBInson, 1998) entre outros fatores. O conhecimento e prognóstico de características da comunidade em resposta a diferentes fatores ambientais é um dos mais importantes objetivos da ecologia de comunidades (ARGERMEIER \& KARR, 1983).

A importância de variáveis ambientais para a comunidade de peixe é dependente da escala de análise. Em pequena escala, fatores bióticos desempenham um papel importante na organização da comunidade. Em estudos de larga escala, no entanto, fatores abióticos e biogeográficos são os determinantes mais importantes das comunidades de peixes (JACKSON et al., 2001).

Comunidades de peixes neotropicais podem ser influenciadas por fatores abióticos (ANGERMEIER \& KARR, 1983) como fotoperíodo (PowER, 1983), condutividade, profundidade, velocidade e volume da água (UIEDA \& Barreto, 1999). A maioria dos estudos visando quantificar os efeitos dos fatores ambientais em comunidades de peixes são desenvolvidos em regiões temperadas (GORMAN \& KARR, 1978; MARTIN-SMITH, 1998; OBERDORFF et al., 2001). Poucos são os estudos em regiões tropicais, especialmente no Neotrópico, como os estudos
De Garutti (1988), Mazzoni \& Lobón-Cerviá (2000), Abes \& Agostinho (2001), Layman \& Winemiller (2005), Arrington \& Winemiller (2006) e Súarez \& Petrere Jr. (2006). Dentre os estudos sobre variações espaciais e temporais em comunidades de peixes neotropicais em lagos destacam-se RodríguEZ \& Lewis (1994), GALACATOS et al. (1996), HeNderson \& Crampton (1997), TeJerinaGarro et al. (1998) e Vono \& Barbosa (2001). Estudos investigando a ictiofauna de lagoas salientam-se os de Handerson \& Hamilton (1995), Meschiatti et al. (2000) e GaLACATOS et al. (2004).

O estudo de comunidades de peixes envolve uma síntese de fatores ambientais e de interações bióticas, podendo a estrutura de uma comunidade ser analisada sob vários aspectos (ARGERMEIER \& KARR, 1983). Dentre os padrões mais abordados em ecologia de comunidades estão aqueles relacionados à diversidade de espécies (número, identidade e as características das mesmas), como também aqueles relativos às variações temporais de suas abundâncias (Wootton, 1990).

O presente estudo visa caracterizar e comparar a taxocenose de peixes de dois ambientes aquáticos distintos, dentro de uma mesma bacia hidrográfica: a praia das Pombas e a lagoa Negra, numa escala espaçotemporal. Visa ainda investigar se ocorre relação entre a estrutura da taxocenose destes ambientes e as variáveis ambientais. 


\section{MATERIAL E MÉTODOS}

Área de estudo. O Parque Estadual de Itapuã ocupa uma área de 5.566 ha, estando localizado a $57 \mathrm{Km}$ ao sul de Porto Alegre, no distrito de Itapuã, município de Viamão, Rio Grande do Sul. O Parque é uma reserva ecológica criada em 1973, representando a última amostra dos ecossistemas e paisagens originais dos campos, dunas, lagos, lagoas, ilhas, praias e morros às margens do lago Guaíba e da laguna dos Patos. Os ecossistemas hídricos do Parque Estadual de Itapuã inserem-se no sistema hidrográfico da laguna dos Patos. O clima local é o subtropical úmido ( $\mathrm{Cfa}$ ), com chuvas bem distribuídas ao longo do ano e verões quentes (KöPPEN, 1948). O bom índice de conservação dos recursos naturais do Parque Estadual de Itapuã, juntamente com a diversidade de ambientes aquáticos da área, permite a preservação de parte considerável da diversidade de peixes do sistema da laguna dos Patos, sendo sua proteção um dos objetivos do Plano de Manejo do Parque (RIo GRANDE Do Sul, 1997).

Praia das Pombas. A localidade praia das Pombas (Fig.1) apresenta forma de baía e é banhada pelo lago Guaíba, um dos mais importantes recursos hidrográficos do Rio Grande do Sul, estendendo-se desde o delta do rio Jacuí, ao norte, até o Pontal de Itapuã, ao sul (MENEGAT et al., 1998). O ponto de coleta situa-se próximo ao trapiche da praia das Pombas $\left(30^{\circ} 20^{\prime} 44,6^{\prime \prime}\right.$ 'S; $\left.51^{\circ} 01^{\prime} 32,6^{\prime \prime} \mathrm{W}\right)$ e é caracterizado por apresentar águas claras, fundo arenoso e profundidade média de 1,5 metros. A vegetação das margens é de mata de restinga identificada principalmente pelos sarandis (Cephalanthus glabratus, Rubiaceae), corticeiras-do-banhado (Erythrina cristagalli, Fabaceae), maricás (Mimosa bimucronata, Fabaceae) e juncos (Cyperaceae: Cyperus spp. e Scirpus spp.).

Lagoa Negra. Esta lagoa ocupa uma área de 1.750 ha e está separada da laguna dos Patos por um terreno arenoso constituído de dunas cobertas por vegetação (Grosser \& HAHN, 1981). Este ambiente não é totalmente isolado, pois ocorre comunicação entre a lagoa Negra e a laguna dos Patos quando as chuvas são muito intensas. De acordo com o Plano de Manejo do Parque (Rio Grande

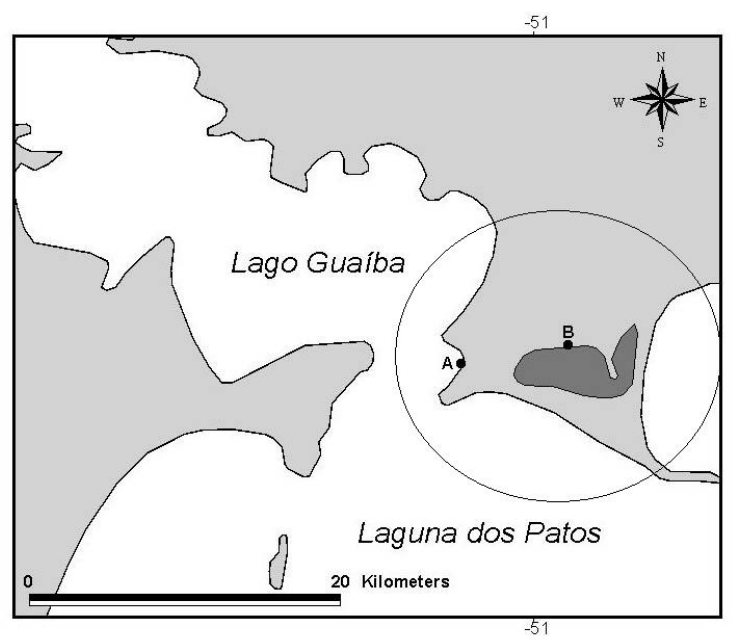

Figura 1. Mapa mostrando os locais de coleta no Parque Estadual de Itapuã, RS (elipse delimitando a área): (A, praia das Pombas; B, lagoa Negra).
Do SuL, 1997), as principais características ambientais da lagoa Negra são suas águas estáveis, fundo arenoso e lodoso, grande diversidade de plantas aquáticas e profundidades de até três metros. Estudos limnológicos sobre a lagoa Negra foram realizados por VoLKMERRiBEIRo (1981), sendo esta assinalada como a única lagoa de águas escuras da Planície Costeira do Rio Grande do Sul.

O ponto de coleta (Fig. 1) está localizado na margem leste da lagoa $\left(30^{\circ} 21^{\prime} 35,5^{\prime \prime} \mathrm{S} ; 50^{\circ} 58^{\prime} 34^{\prime \prime} \mathrm{O}\right)$ e se caracteriza pela baixa transparência, devido ao elevado teor de matéria orgânica particulada em suspensão na água. A vegetação aquática é formada predominantemente por juncos (Cyperus spp. e Scirpus spp.), em formações esparsas. Junto às margens, encontra-se grande quantidade de macrófitas aquáticas principalmente repolhos-d'água (Pistia stratiotes, Araceae), salvínias (Salvinia sp., Salviniaceae), soldanelas-d'água (Nynphoides indica, Menyanthaceae) e aguapés (Eicchornia spp., Pontederiaceae).

Amostragem. As coletas foram realizadas mensalmente, entre junho de 2002 e julho de 2003, com exceção do mês de dezembro, totalizando 13 amostragens em cada local de coleta. Os indivíduos foram capturados com rede do tipo picaré de $10 \mathrm{~m}$ de comprimento, $2,5 \mathrm{~m}$ de altura e $5 \mathrm{~mm}$ entre nós adjacentes, sendo o esforço de captura de cinco arrastos/ponto/mês. Foi também utilizada uma bateria de redes de espera em cada ponto, com 1,5; 2,$0 ; 2,5 ; 3,0$ e $3,5 \mathrm{~cm}$ entre nós adjacentes $(20 \mathrm{~m}$ de comprimento por $1,5 \mathrm{~m}$ de altura cada rede) as quais foram dispostas a uma distância de aproximadamente dois metros da margem, onde permaneciam durante cinco horas, em cada ponto.

Dados de temperatura da água, temperatura do ar, condutividade, $\mathrm{pH}$ e oxigênio dissolvido foram tomados mensalmente nos locais de coleta, no momento da amostra. Dados pluviométricos foram obtidos junto ao Oitavo Distrito de Meteorologia - Serviço de Observação e Meteorologia Aplicada de Porto Alegre. O horário do nascer e pôr-do-sol e o cálculo do fotoperíodo foram obtidos através do programa de computador SkyMap Pro 9.0, correlacionando as datas às coordenadas do local de coleta.

Os indivíduos capturados foram separados de acordo com o local de coleta e tipo de rede e então fixados em formalina $10 \%$. Em laboratório, os peixes foram transferidos para etanol $70^{\circ} \mathrm{GL}$ e identificados até espécie. De cada indivíduo foram registrados dados de comprimento padrão, em milímetros ( $\mathrm{mm}$ ) e peso total, em gramas $(\mathrm{g})$. Os espécimes testemunho deste estudo estão depositados na Coleção de Peixes do Departamento de Zoologia da Universidade Federal do Rio Grande do Sul, Porto Alegre, Brasil (lotes UFRGS5046; UFRGS5688; UFRGS5701-5703;UFRGS5705; UFRGS5707; UFRGS5709; UFRGS5712-5714;UFRGS5717;UFRGS5719; UFRGS5721; UFRGS5723, 5724; UFRGS5726; UFRGS5729, 5730; UFRGS5732; UFRGS6010;UFRGS6134;UFRGS6695-6702; UFRGS6706-6708; UFRGS6710-6721; UFRGS6723-6725; UFRGS6727-6730; UFRGS6734; UFRGS6736-6738; UFRGS6740; UFRGS7655; UFRGS9777; UFRGS9779; UFRGS9782). 
Análise dos dados. Para verificar se os métodos de coleta empregados foram eficientes para a amostragem das espécies de peixes da praia das Pombas e da lagoa Negra, foi aplicada a curva do coletor, que relaciona o número de espécies acumuladas com as visitas realizadas ao campo, para cada arte de pesca. Segundo CAIN (1938), a suficiência amostral é atingida quando um incremento de $10 \%$ no tamanho da amostra corresponde a um incremento de $10 \%$ ou menos no número de espécies coletadas. Foi também calculada a riqueza de espécies esperada, através do estimador Michaelis-Menten (Colwell, 2000). A curva do coletor e a riqueza de espécies foram calculados através do software EstimateS 7.51.

A abundância de cada espécie foi representada pelo número de indivíduos capturados com ambos os métodos de coleta (picaré + redes de espera). A biomassa foi representada pelo peso total dos indivíduos capturados, em gramas. Para o estudo da variação temporal desses dois parâmetros, os valores foram analisados mensalmente.

Foi calculada a constância de ocorrência (DAJoz, 1978), através da fórmula: $\mathrm{C}=(\mathrm{P} / \mathrm{Q}) \times 100$ onde, $\mathrm{C}=$ constância de ocorrência da espécie; $\mathrm{P}=$ número de meses em que a espécie ocorreu e $\mathrm{Q}=$ número total de meses. As espécies foram classificadas em constantes $(>50 \%)$, acessórias $(25 \geq 50 \%)$ e acidentais $(\leq 25 \%)$. A diversidade de espécies de Shannon \& Wiener (Pielou, 1975) foi calculada com o auxílio do software DIVERS (PÉrEZ-LóPEZ \& Sola-Fernandez, 1993).

A fim de verificar padrões de similaridade temporal entre os pontos de amostragem e os métodos de coleta utilizados, foi aplicada uma análise de cluster através do índice Pearson e o método de aglomeração UPGMA, utilizando a abundância absoluta das espécies de cada amostra. Para verificar a similaridade na composição das espécies entre os dois locais de amostragem e os métodos de coleta, foi aplicado um teste de dissimilaridade através da distância de Bray-Curtis e o método de aglomeração de Ward.

Para testar a influência dos fatores ambientais (temperatura do ar e da água, pluviosidade, fotoperíodo, $\mathrm{pH}$, condutividade e oxigênio dissolvido) sobre a abundância, biomassa e diversidade foi aplicada o teste não-paramétrico de regressões múltiplas com análise de variância (ANOVA; ZAR, 1999) aos dados mensais.

Aos dados de abundância mensal das espécies que ocorreram nos dois ambientes, por arte de pesca, e os fatores ambientais foi aplicada uma análise de correspondência canônica (ACC; TER BrAAK, 1986). Este é um dos melhores métodos para análise direta de gradientes em ecologia de comunidades por descrever a tendência principal entre a distribuição de espécies e os fatores ambientais correlatos (TER BRAAK, 1986; RoDRIGUEZ \& LEWIS, 1997). Uma análise de componentes principais (ACP) foi aplicada aos dados de abundância mensal das espécies coletadas nas redes de espera na praia das Pombas, a fim de verificar se a alta abundância de Cyphocharax voga no mês de junho de 2002 foi significativa.

Os testes estatísticos utilizados neste estudo foram realizados através do programa XLSTAT Versão 2008.6.05.

\section{RESULTADOS}

Praia das Pombas. Foram capturados 2.160 exemplares de peixes, 1.600 com picaré e 560 com as redes de espera. Estes indivíduos foram distribuídos em seis ordens, 17 famílias e 44 espécies (Tab. I). O comprimento padrão das espécies capturadas variou entre 12,8 e 290 $\mathrm{mm}$. A curva do coletor obtida para cada arte de pesca, através das aleatorizações geradas a partir dos dados obtidos em campo mostra um aumento no número de espécies para cada coleta (Fig. 2). A curva não atingiu um platô horizontal para as espécies capturadas com o picaré, pois foi capturada uma nova espécie na última amostragem. No entanto, a curva das redes de espera estabilizou a partir da oitava amostragem. A riqueza de espécies esperada para ambos os métodos, obtida através do estimador Michaelis-Menten, mostrou que $82 \%$ (39) das espécies esperadas para o picaré neste ambiente foram amostradas. Já para as redes de espera, foram amostradas $90 \%$ (26) das espécies estimadas para este ambiente.

As ordens Characiformes e Siluriformes foram as mais representativas em número de espécies, correspondendo a $36,4 \%$ (16 espécies) e $27,3 \%$ (12 espécies), respectivamente. Do total de indivíduos capturados, cinco espécies corresponderam a 79,1\%: Cyanocharax alburnus (Hensel, 1870), Astyanax fasciatus (Cuvier, 1819), Cyphocharax voga (Hensel, 1870), Pachyurus bonariensis Steindachner, 1879 e Astyanax jacuhiensis Cope, 1894 (Tab. I). Oito espécies contribuíram com 83,5\% em peso: C. voga, P. bonariensis, Loricariichthys anus (Valenciennes, 1836), Pimelodus maculatus (La Cépède, 1803), Crenicichla punctata (Hensel, 1870), A. fasciatus, Lycengraulis grossidens (Spix \& Agassiz, 1892) e A. jacuhiensis.

A frequência de ocorrência das espécies capturadas durante o período estudado, analisando picaré e redes de espera juntamente, mostrou que 13 espécies foram consideradas constantes (Tab. I). Destas, A. fasciatus, $C$. alburnus e Rineloricaria strigilata (Hensel, 1868) ocorreram em todos os meses de amostragem. Analisando a variação da abundância durante os meses de amostragem (Fig. 3) observou-se que as maiores abundâncias ocorreram nos meses de setembro de 2002 e janeiro de 2003.

A biomassa total dos indivíduos capturados com o picaré e com as redes de espera foi $3.631 \mathrm{~g} \mathrm{e} 29.104 \mathrm{~g}$, respectivamente, totalizando $32.735 \mathrm{~g}$. Foi observado um aumento na biomassa nos meses de junho e outubro de 2002 (Fig. 4). O elevado valor da biomassa em junho deve-se à captura expressiva de $C$. voga $(8.624,60 \mathrm{~g})$ neste mês. Uma análise de componentes principais (ACP) foi aplicada aos valores mensais de abundância das espécies capturadas nas redes de espera. Foi observado que C. voga contribuiu com $86,5 \%$ das variações em dois eixos (F1 $=40,238$ e F2 $=46,260)$ e que a abundância desta espécie está correlacionada ao mês de junho de 2002 .

A diversidade de espécies no picaré e nas redes foram semelhantes (1,9 e 2,0; respectivamente). A distribuição mensal da diversidade mostrou, através na análise de variância, dependência significativa com a pluviosidade $(\mathrm{t}=-3,161$ e $\mathrm{Pr}=0,012)$ e fotoperíodo $(\mathrm{t}=3,262$ e $\operatorname{Pr}=0,01)$ para as espécies do picaré e com a condutividade ( $\mathrm{t}=2,832$ e $\mathrm{Pr}=0,02)$ e fotoperíodo ( $\mathrm{t}=1,898$ e $\operatorname{Pr}=0,09$ ) para as redes de espera. 
Praia das Pombas

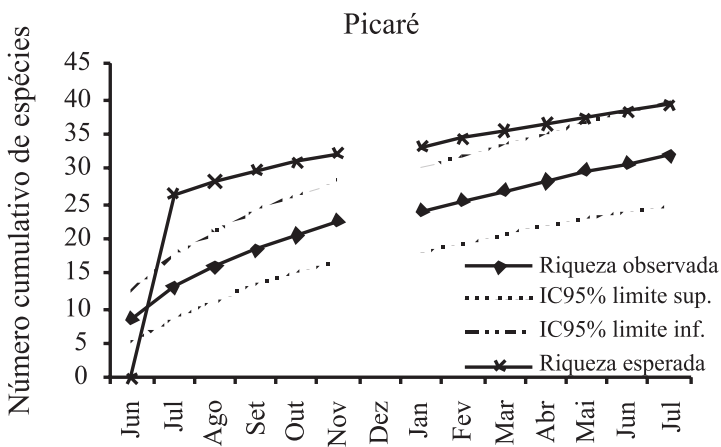

Redes de espera

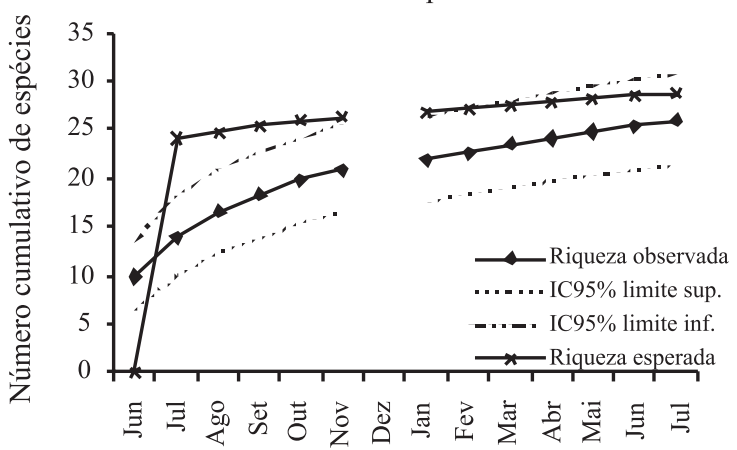

Lagoa Negra

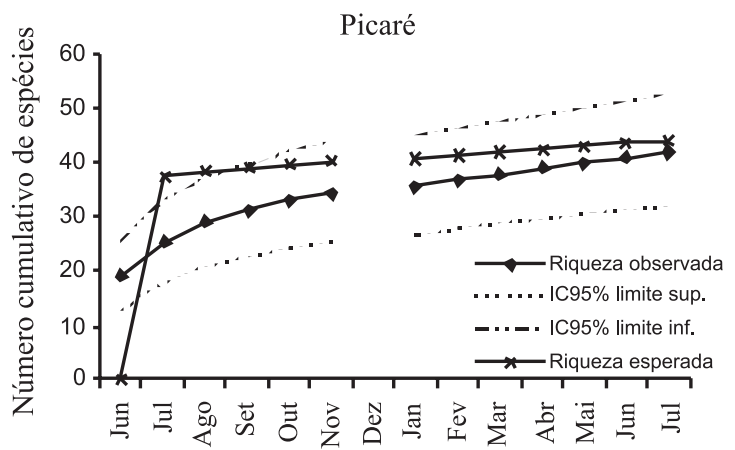

Redes de espera

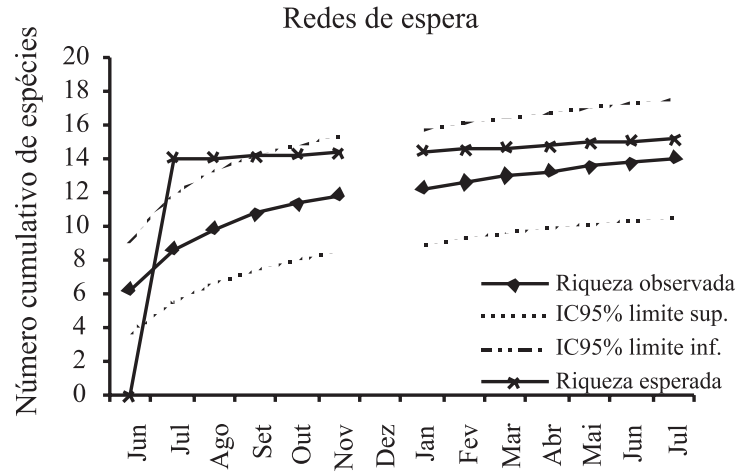

Figura 2. Curva do coletor obtida a partir das amostragens de peixes realizadas na praia das Pombas e na lagoa Negra, Parque Estadual de Itapuã, Viamão, RS, no período entre junho de 2002 a julho de 2003, com picaré e redes de espera.

3 Os clusters de abundância de espécies (Pearson)
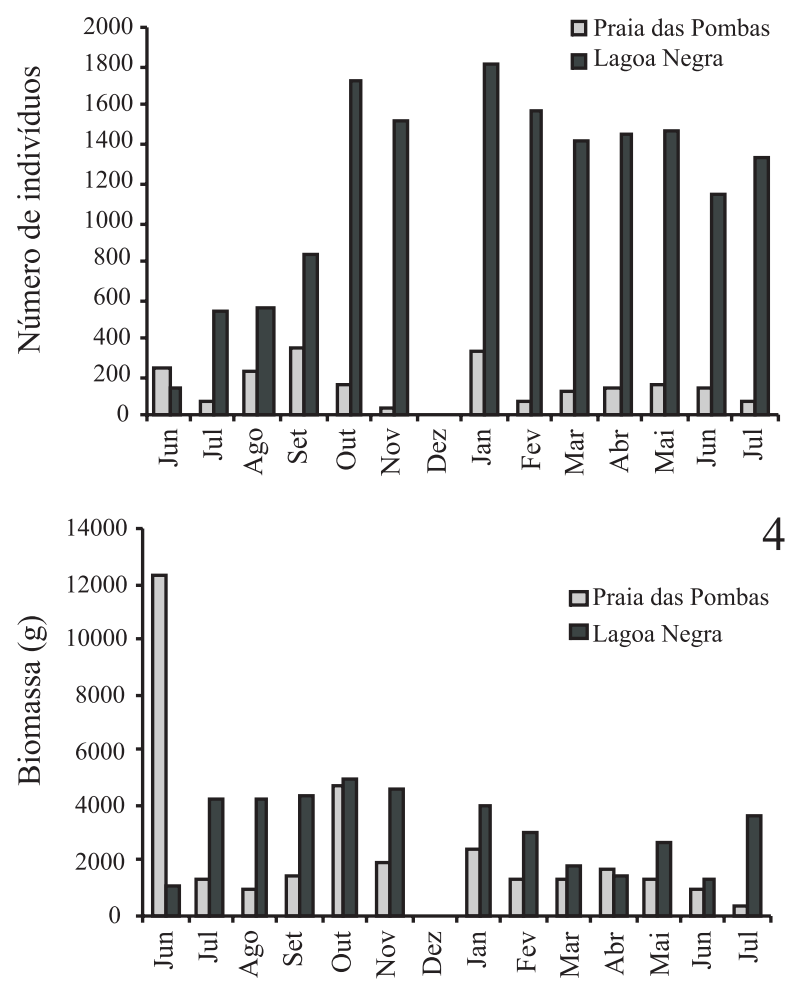

Figuras 3, 4. Variação mensal total, para a taxocenose de peixes da praia das Pombas e da lagoa Negra, Parque Estadual de Itapuã, Viamão, RS, entre junho de 2002 e julho de 2003: 3, abundância (número de indivíduos); 4, biomassa (gramas). do picaré e das redes de espera separaram claramente os meses de maior temperatura dos de menor temperatura (Fig. 5), mostrando claramente a distribuição sazonal das espécies na praia das Pombas.

Lagoa Negra. Do total de 13 amostragens realizadas, foram capturados 15.557 indivíduos, sendo 15.240 coletados com picaré e 321 com redes de espera. Estes exemplares estão distribuídos em oito ordens, 18 famílias e 44 espécies (Tab. I). A variação do comprimento padrão entre os indivíduos foi de 9,1 a $410 \mathrm{~mm}$. Neste ambiente, as redes de espera foram mais eficientes, segundo a curva do coletor (Fig. 2), pois houve uma adição de espécies à amostra coletada através do picaré nas últimas coletas. As riquezas esperadas para o picaré e para as redes de espera foram altas, $98 \%$ (44) e 100\% (14), respectivamente.

Igualmente à praia das Pombas, na lagoa Negra as ordens Characiformes e Siluriformes foram as mais representativas quanto ao número de espécies, correspondendo a 40,9\% (18 espécies) e 29,5\% (13 espécies), respectivamente. Do total de indivíduos amostrados, seis espécies perfizeram $89,8 \%$ do número de indivíduos capturados: Cheirodon ibicuhiensis Eigenmann, 1915, C. alburnus, Hyphessobrycon luetkenii (Boulenger, 1887), Cheirodon interruptus (Jenyns, 1842), Hyphessobrycon bifasciatus Ellis, 1911 e Astyanax eigenmanniorum (Cope, 1894) (Tab. I). Em peso, nove espécies participaram com $87,8 \%$ do total: C. voga, Hoplias malabaricus (Bloch, 1794), P. maculatus, Hypostomus commersoni Valenciennes, 1836, Hoplosternum littorale (Hancock, 1828), Oligosarcus robustus Menezes, 1969, Geophagus brasiliensis (Quoy \& Gaimard, 1824), C. ibicuhiensis e L. anus. 
Analisando a freqüência de ocorrência das espécies capturadas no período de estudo verificou-se que 21 foram consideradas constantes. Destas, Astyanax jacuhiensis Cope, 1894, A. eigenmanniorum, C. alburnus, C. ibicuhiensis, C. voga, G. brasiliensis, $H$. bifasciatus e $H$. luetkenii ocorreram em todos os meses de coleta. Através da análise da abundância total, analisando picaré e rede de espera em conjunto, pode-se observar que as maiores abundâncias foram registradas nos meses de outubro de 2002 e janeiro de 2003 (Fig. 3). A biomassa obtida na lagoa Negra através do picaré foi de 13.990 g e com as redes de espera $27.477 \mathrm{~g}$, totalizando assim, $41.536 \mathrm{~g}$. Esses valores foram mais elevados nos meses de outubro e novembro de 2002 (Fig. 4).
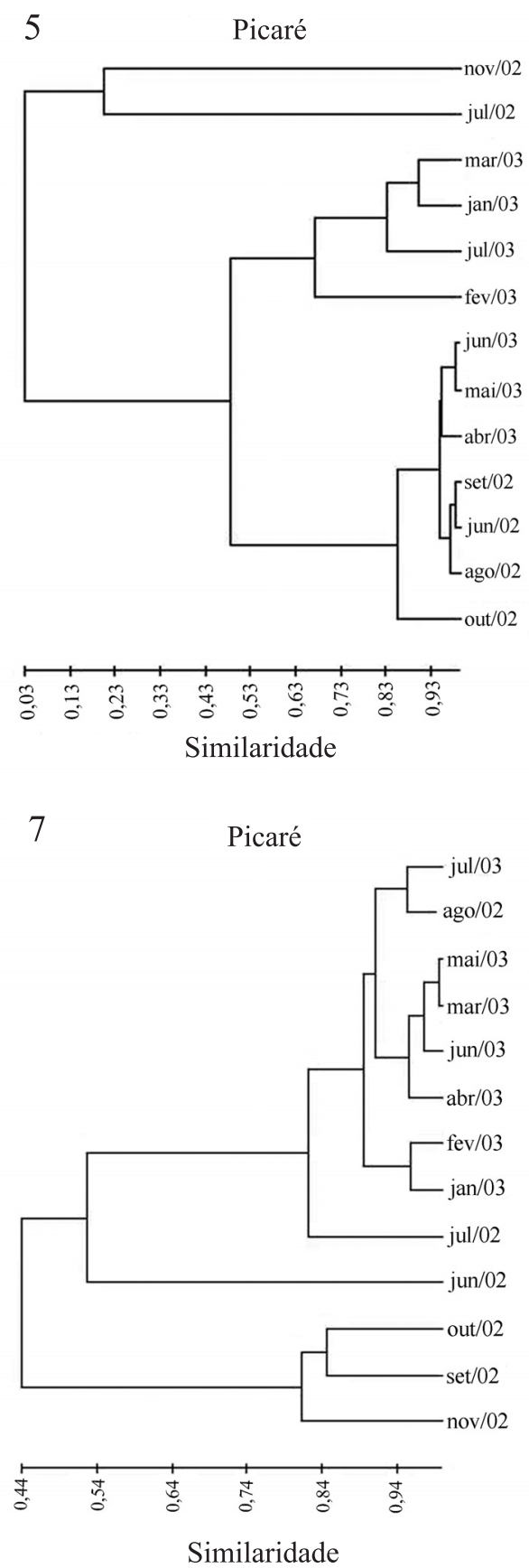

A diversidade encontrada no picaré foi menor do que nas redes de espera $\left(H^{\prime}=1,6\right.$ e $H^{\prime}=2,2$, respectivamente). A análise de variância (ANOVA), através de regressões múltiplas, mostrou que a diversidade mensal do picaré é significativamente dependente do $\mathrm{pH}(\mathrm{t}=2,574$ e $\mathrm{Pr}=0,028)$ e fotoperíodo $(t=3,378$ e $\operatorname{Pr}=0,007)$ enquanto que, para as redes de espera, a diversidade apresenta dependência da pluviosidade $(\mathrm{t}=2,415$ e $\operatorname{Pr}=0,036)$ e do fotoperíodo $(\mathrm{t}=1,365$ e $\mathrm{Pr}=0,202)$.

A análise de cluster através do coeficiente de similaridade de Pearson mostrou que a distribuição das espécies de peixes coletadas, tanto com picaré quanto com redes de espera foi sazonal (Figs. 5-8).

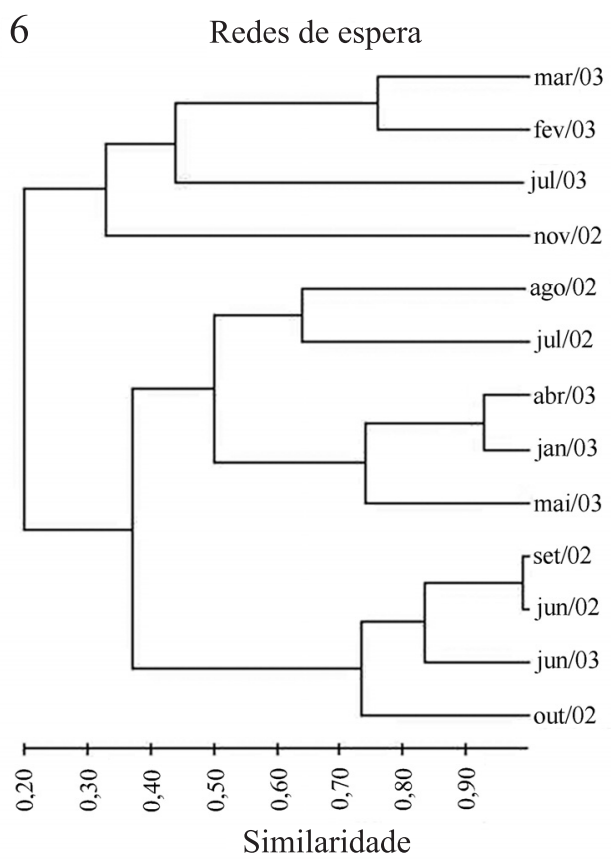

8
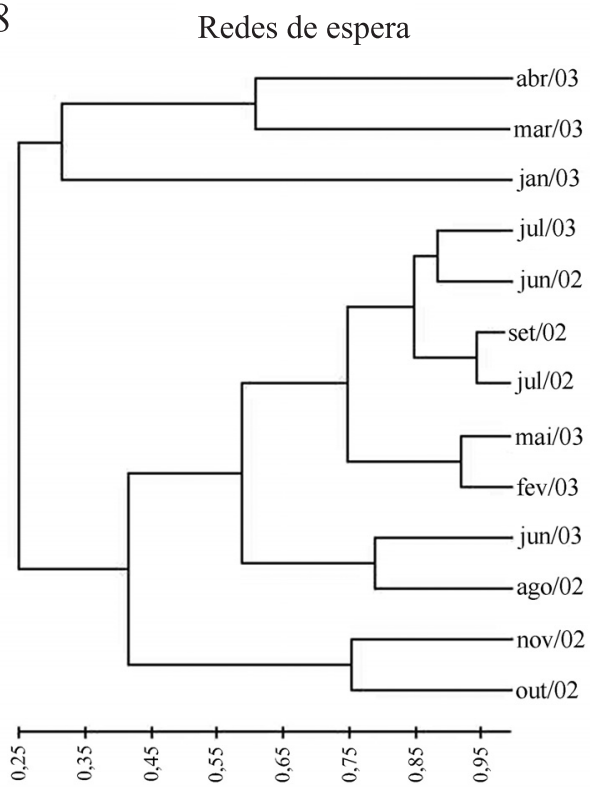

Similaridade

Figuras 5-8. Clusters de similaridade (coeficiente de Pearson) da taxocenose de peixes no Parque Estadual de Itapuã, Viamão, RS: 5, 6, praia das Pombas; 7, 8, lagoa Negra. 
Tabela I. Lista das espécies de peixes registradas para a praia das Pombas e lagoa Negra, Parque Estadual de Itapuã, Viamão, RS, com suas abundâncias no picaré e redes de espera (AC, acidental; AS, acessória; C, constância de ocorrência; CO, constante; ${ }^{a}$, presente estudo; b, LuCena et al., 1994; ' , Grosser \& Hahn, 1981; " 1 , gênero, provavelmente, identificado erroneamente; ${ }^{* 2}$, listada como Characidium fasciatum por ${ }^{\mathrm{b}}$; ${ }^{* 3}$, listada como Schizodon fascitatus por ${ }^{c}$; ${ }^{* 4}$, espécie de taxonomia confusa, nome científico incorreto; ${ }^{* 5}$, gênero com taxonomia confusa e os limites entre as diferentes espécies são confusos. A espécie listada como $R$. sapo por ${ }^{\mathrm{c}}$ pode ser a mesma referida como Rhamdia quelen neste mesmo trabalho; ${ }^{* 6}$, listada como Crenicichla saxatilis por ${ }^{c} ;{ }^{* 7}$, listada como Jenynsia lineata por b, c ; ${ }^{*}$, listada como Eigenmannia virescens por ${ }^{\mathrm{c}}$ ).

\begin{tabular}{|c|c|c|c|c|c|c|}
\hline \multirow[t]{2}{*}{ Táxons } & \multicolumn{3}{|c|}{$\begin{array}{l}\text { Praia das Pombas } \\
\text { Número de indivíduos }\end{array}$} & \multicolumn{3}{|c|}{$\begin{array}{l}\text { Lagoa Negra } \\
\text { Número de indivíduos }\end{array}$} \\
\hline & $\mathrm{C}$ & Redes espera & Picaré & $\mathrm{C}$ & Redes espera & Picaré \\
\hline \multicolumn{7}{|l|}{ CHARACIFORMES } \\
\hline \multicolumn{7}{|l|}{ Characidae } \\
\hline Aphyocharax anisitsi Eigenmann \& Kennedy, 1903ª & $\mathrm{AC}$ & & 1 & $\mathrm{CO}$ & & 18 \\
\hline Astyanax eigenmanniorum (Cope, 1894) $)^{\mathbf{a}, \mathbf{b}, \mathbf{c}}$ & & & & $\mathrm{CO}$ & & 1056 \\
\hline Astyanax fasciatus (Cuvier, 1819), $\mathbf{a}, \mathbf{b}, \mathbf{c}$ & $\mathrm{CO}$ & 69 & 275 & $\mathrm{AC}$ & 3 & \\
\hline Astyanax jacuhiensis Cope, $1894^{\mathbf{a}, \mathbf{b}, \mathbf{c}}$ & $\mathrm{CO}$ & 37 & 59 & $\mathrm{CO}$ & 30 & 258 \\
\hline \multicolumn{7}{|l|}{ Astyanax scabripinnis (Jenyns, 1842) ${ }^{\mathrm{c}}$} \\
\hline Astyanax sp. ${ }^{\mathrm{a}}$ & & & & AS & & 37 \\
\hline Astyanax sp. $\mathrm{c}^{\mathrm{a}}$ & & $\mathrm{AC}$ & & 2 & & \\
\hline Bryconamericus iheringii (Boulenger, 1887), b & $\mathrm{AC}$ & & 3 & $\mathrm{AC}$ & & 1 \\
\hline \multicolumn{7}{|l|}{ Charax stenopterus (Cope, 1894) ${ }^{\mathbf{C}}$} \\
\hline Cheirodon ibicuhiensis Eigenmann, 1915 a & $\mathrm{AC}$ & & 1 & $\mathrm{CO}$ & & 6282 \\
\hline Cheirodon interruptus (Jenyns, 1842) & & & & $\mathrm{CO}$ & & 1148 \\
\hline Cyanocharax alburnus (Hensel, 1870) ${ }^{\mathbf{a}, \mathbf{b}}$ & $\mathrm{CO}$ & & 915 & $\mathrm{CO}$ & & 3003 \\
\hline \multicolumn{7}{|l|}{ Deuterodon sp. ${ }^{\mathrm{c},{ }^{* 1}}$} \\
\hline Hyphessobrycon anisitsi (Eigenmann, 1907) ${ }^{\mathrm{a}}$ & $\mathrm{AC}$ & 3 & & & & \\
\hline Hyphessobrycon bifasciatus Ellis, 1911 a, c & & & & $\mathrm{CO}$ & & 1099 \\
\hline Hyphessobrycon boulengeri (Eigenmann, 1907)a, c & & & & AS & & 57 \\
\hline Hyphessobrycon luetkenii (Boulenger, 1887)a, b, c & $\mathrm{AC}$ & 1 & 5 & $\mathrm{CO}$ & & 1381 \\
\hline Oligosarcus jenynsii (Günther, 1864) $)^{\mathbf{a}, \mathbf{b}, \mathbf{c}}$ & $\mathrm{AC}$ & 6 & & $\mathrm{CO}$ & 39 & 6 \\
\hline Oligosarcus robustus Menezes, $1969^{\mathbf{a}, \mathbf{b}, \mathbf{c}}$ & $\mathrm{CO}$ & 7 & 12 & $\mathrm{CO}$ & 31 & 25 \\
\hline Pseudocorynopoma doriae Perugia, $1891^{\mathbf{a}, \mathbf{b}, \mathbf{c}}$ & & & & $\mathrm{CO}$ & & 112 \\
\hline Serrapinnus calliurus (Boulenger, 1900) ${ }^{\mathrm{a}}$ & $\mathrm{AC}$ & & 3 & $\mathrm{AC}$ & & 1 \\
\hline \multicolumn{7}{|l|}{ Crenuchidae } \\
\hline \multicolumn{7}{|l|}{ Characidium tenue (Cope, 1984) } \\
\hline \multicolumn{7}{|l|}{ Characidium zebra Eigenmann, $1909^{\mathrm{c},{ }^{* 2}}$} \\
\hline \multicolumn{7}{|l|}{ Curimatidae } \\
\hline Cyphocharax saladensis (Meinken, 1933) ) $^{\mathbf{a}, \mathbf{c}}$ & & & & $\mathrm{AC}$ & & 2 \\
\hline Cyphocharax voga $($ Hensel, 1870) a, b, c & $\mathrm{CO}$ & 207 & 46 & $\mathrm{CO}$ & 110 & 63 \\
\hline \multicolumn{7}{|l|}{ Anostomidae } \\
\hline Leporinus obtusidens (Valenciennes, 1836) ${ }^{\mathrm{a}}$ & AS & 4 & & & & \\
\hline Schizodon jacuiensis Bergman, $1988^{\mathrm{a}, \mathrm{c},{ }^{*}}$ & $\mathrm{AC}$ & 3 & & & & \\
\hline \multicolumn{7}{|l|}{ Erythrinidae } \\
\hline Hoplias malabaricus (Bloch, 1794), a, b, c & $\mathrm{AC}$ & 3 & & $\mathrm{CO}$ & 31 & 58 \\
\hline \multicolumn{7}{|l|}{ Lebiasinidae } \\
\hline Pyrrhulina australis Eigenmann \& Kennedy, $1903^{\mathbf{c}}$ & & & & & & \\
\hline SILURIFORMES & & & & & & \\
\hline Aspredinidae & & & & & & \\
\hline Ancistrus brevipinnis (Regan, 1904) ${ }^{\mathbf{a}}$ & $\mathrm{AC}$ & 1 & & & & \\
\hline Bunocephalus sp. ${ }^{\mathrm{a}}$ & $\mathrm{AC}$ & & 1 & & & \\
\hline Bunocephalus iheringii Boulenger, $1891^{\mathbf{a}, \mathbf{c}}$ & & & & AS & & 9 \\
\hline Auchenipteridae & & & & & & \\
\hline Glanidium albescens Lütken, $1874^{\mathrm{c},{ }^{* 4}}$ & & & & & & \\
\hline Callichthyidae & & & & & & \\
\hline Corydoras paleatus (Jenyns, 1842) a, b, c & $\mathrm{AC}$ & & 1 & $\mathrm{CO}$ & & 38 \\
\hline Callichthys callichthys (Linnaeus, 1758) ${ }^{\mathbf{c}}$ & & & & & & \\
\hline Hoplosternum littorale (Hancock, 1828) $)^{\mathbf{a}, \mathbf{b}}$ & & & & AS & 12 & 1 \\
\hline Heptapteridae & & & & & & \\
\hline Heptapterus mustelinus (Valenciennes, 1835) ${ }^{\mathrm{c}}$ & & & & & & \\
\hline Pimelodella australis Eigenmann, 1917 a, b, c & & & & $\mathrm{AC}$ & & 16 \\
\hline Rhandella sp. ${ }^{\mathrm{c}}$ & & & & & & \\
\hline Rhamdia sp. ${ }^{\mathrm{a}, \mathrm{c}, * 5}$ & & & & $\mathrm{AC}$ & & 2 \\
\hline Loricariidae & & & & & & \\
\hline Hisonotus nigricauda, $a^{\mathbf{a}, \mathbf{b}, \mathbf{c}}$ & $\mathrm{AC}$ & & 1 & $\mathrm{CO}$ & & 138 \\
\hline Hypostomus commersoni Valenciennes, $1836^{\mathbf{a}, \mathbf{b}}$ & $\mathrm{AC}$ & & 1 & $\mathrm{CO}$ & 2 & 33 \\
\hline $\begin{array}{l}\text { Loricariichthys anus (Valenciennes, 1836) } \\
\text { Otothyris } \mathbf{b}, \mathbf{c} \\
\text { sp }\end{array}$ & $\mathrm{CO}$ & 26 & 1 & AS & 4 & 1 \\
\hline Rineloricaria cadeae (Hensel, 1868) $)^{\mathbf{a}, \mathbf{b}}$ & $\mathrm{CO}$ & & 19 & $\mathrm{AC}$ & & 2 \\
\hline Rineloricaria sp. ${ }^{\mathrm{a}}$ & $\mathrm{AC}$ & & 1 & & & \\
\hline Rineloricaria strigilata $(\text { Hensel, 1868) })^{\mathbf{a}, \mathbf{b}}$ & $\mathrm{CO}$ & 7 & 58 & $\mathrm{AC}$ & & 1 \\
\hline
\end{tabular}




\begin{tabular}{|c|c|c|c|c|c|c|}
\hline \multicolumn{7}{|l|}{ Tabela I (cont.) } \\
\hline \multicolumn{7}{|l|}{ Pimelodidae } \\
\hline Parapimelodus nigribarbis (Boulenger, 1889)a, b & $\mathrm{AC}$ & 8 & 14 & & & \\
\hline Pimelodus maculatus (La Cépède, 1803)a, b, c & $\mathrm{CO}$ & 25 & 21 & $\mathrm{CO}$ & 40 & 1 \\
\hline \multicolumn{7}{|l|}{ Pseudopimelodidae } \\
\hline Microglanis cottoides (Boulenger, 1891) a, $^{\mathrm{c}}$ & & & & AS & & 5 \\
\hline \multicolumn{7}{|l|}{ Trichomycteridae } \\
\hline Homodiaetus anisitsi Eigenmann \& Word, 1907a, b & $\mathrm{CO}$ & & 53 & $\mathrm{AC}$ & & 1 \\
\hline \multicolumn{7}{|l|}{ PERCIFORMES } \\
\hline \multicolumn{7}{|l|}{ Cichlidae } \\
\hline \multicolumn{7}{|l|}{ Australoheros facetum (Jenyns, 1842$)^{\mathrm{c}}$} \\
\hline Cichlasoma portalegrense (Hensel, 1870) a, c $^{2}$ & & & & $\mathrm{CO}$ & 1 & 28 \\
\hline Crenicichla lepidota Heckel, $1840^{\mathrm{a}, \mathrm{c}}$ & $\mathrm{AC}$ & 4 & & AS & 3 & 6 \\
\hline Crenicichla punctata Hensel, $1870^{\mathrm{a}, \mathrm{b}, \mathrm{c}}$ & AS & 24 & 2 & & & \\
\hline Geophagus brasiliensis (Quoy \& Gaimard, 1824)a, b, c & $\mathrm{CO}$ & 10 & & AS & 3 & 6 \\
\hline Gymnogeophagus gymnogenys (Hensel, 1870) & AS & 1 & 4 & $\mathrm{AC}$ & & 2 \\
\hline Gymnogeophagus labiatus (Hensel, 1870) ${ }^{\mathrm{a}, \mathrm{b}}$ & AS & 4 & 1 & & & \\
\hline Gymnogeophagus rhabdotus (Hensel, 1870) ${ }^{\mathrm{a}}$ & $\mathrm{AC}$ & 1 & & & & \\
\hline \multicolumn{7}{|l|}{ Gobidae } \\
\hline Ctenogobius shufeldti (Jordan \& Eigenmann, 1887)a, b, c & $\mathrm{AC}$ & & 1 & & & \\
\hline \multicolumn{7}{|l|}{ Mugilidae } \\
\hline \multicolumn{7}{|l|}{ Mugil platanus Günther, $1880^{\mathrm{b}}$} \\
\hline \multicolumn{7}{|l|}{ Sciaenidae } \\
\hline \multicolumn{7}{|l|}{ Micropogonias furnieri (Desmarest, 1983) ${ }^{\mathrm{b}}$} \\
\hline Pachyurus bonariensis Steindachner, $1879^{\mathrm{a}}$ & $\mathrm{CO}$ & 82 & 19 & & & \\
\hline \multicolumn{7}{|l|}{ CLUPEIFORMES } \\
\hline \multicolumn{7}{|l|}{ Clupeidae } \\
\hline Platanichthys platana (Regan, 1917) & AS & & 7 & $\mathrm{AC}$ & & 169 \\
\hline \multicolumn{7}{|l|}{ Engraulidae } \\
\hline Lycengraulis grossidens (Spix \& Agassiz, 1892) & $\mathrm{CO}$ & 22 & 18 & & & \\
\hline \multicolumn{7}{|l|}{ ATHERINIFORMES } \\
\hline \multicolumn{7}{|l|}{ Atherinopsidae } \\
\hline Odontesthes sp. (jovens) ${ }^{\mathrm{a}}$ & $\mathrm{AC}$ & & 52 & & & \\
\hline Odontesthes humensis de Buen, $1953^{\mathrm{a}, \mathrm{b}}$ & $\mathrm{AC}$ & 1 & & $\mathrm{AC}$ & 1 & \\
\hline Odontesthes mirinensis Bemvenuti, $1996^{\mathrm{a}}$ & $\mathrm{AC}$ & 1 & & & & \\
\hline \multicolumn{7}{|l|}{ Odontesthes perugiae Evermann \& Kendall, $1906^{\mathrm{b}}$} \\
\hline Odontesthes sp. ${ }^{\text {a }}$ & $\mathrm{AC}$ & 3 & & & & \\
\hline \multicolumn{7}{|l|}{ CYPRINODONTIFORMES } \\
\hline Anablepidae & & & & & & \\
\hline Jenynsia multidentata (Jenyns, 1842) a, b, c, *7 & $\mathrm{AC}$ & & 2 & AS & & 8 \\
\hline Poeciliidae & AC & & 2 & AD & & \\
\hline Phalloceros caudimaculatus (Hensel, 1868) & $\mathrm{AC}$ & & 1 & $\mathrm{CO}$ & & 52 \\
\hline Rivulidae & AC & & 1 & $c 0$ & & \\
\hline Cynopoecilus melanotaenia (Regan, 1912) ), $^{\mathrm{a}, \mathrm{c}}$ & & & & $\mathrm{AC}$ & & 4 \\
\hline GYMNOTIFORMES & & & & & & \\
\hline Gymnotidae & & & & & & \\
\hline Gymnotus sp. & & & & & & \\
\hline Hypopomidae & & & & & & \\
\hline Brachyhypopomus sp. ${ }^{\mathrm{a}}$ & & & & $\mathrm{AC}$ & & 1 \\
\hline Sternopygidae & & & & & & \\
\hline Eigenmannia trilineata López \& Castello, $1966^{\text {a, c, }{ }^{* 8}}$ & & & & $\mathrm{CO}$ & & 19 \\
\hline SYNBRANCHIFORMES & & & & & & \\
\hline Synbranchidae & & & & & & \\
\hline Synbranchus marmoratus Bloch, $1795^{\mathrm{a}, \mathrm{c}}$ & & & & $\mathrm{AC}$ & & 1 \\
\hline
\end{tabular}

A análise de cluster realizada para verificar a similaridade entre os dois ambientes mostrou que a composição de espécies coletadas no picaré na praia das Pombas é pouco similar à composição de espécies da lagoa Negra (distância de Bray-Curtis $=0,875$ ), coletadas com este mesmo método. No entanto, esta distância foi mais baixa comparando-se as espécies coletadas nas redes de espera entre os dois ambientes $(0,544)$.

A análise de variância (ANOVA), através de regressões múltiplas, mostrando as relações entre os diferentes parâmetros obtidos da taxocenose com os fatores ambientais. $\mathrm{O}$ fotoperíodo foi o principal fator que influenciou na abundância e na biomassa obtida através do picaré nos dois ambientes estudados. Já o oxigênio dissolvido teve mais influência sobre a abundância e a biomassa das redes de espera (Tab. II).

A ACC foi aplicada às espécies que apresentaram maior abundância nos locais amostrados, mostrando os fatores ambientais mais importantes para determinar a distribuição das espécies de peixes nos dois ambientes, de acordo como método de coleta empregado (Tab. III, Figs. 9, 10). A maior parte da variação foi explicada pelas variáveis ambientais na praia das Pombas $(68,04 \%$ para o picaré e $77,03 \%$ para as redes de espera) e na lagoa Negra 


\section{Picaré}

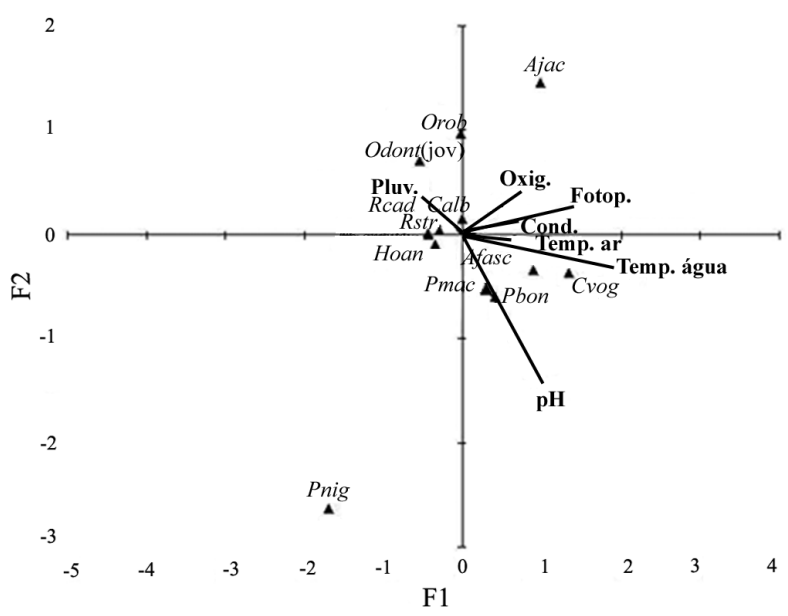

Redes de espera

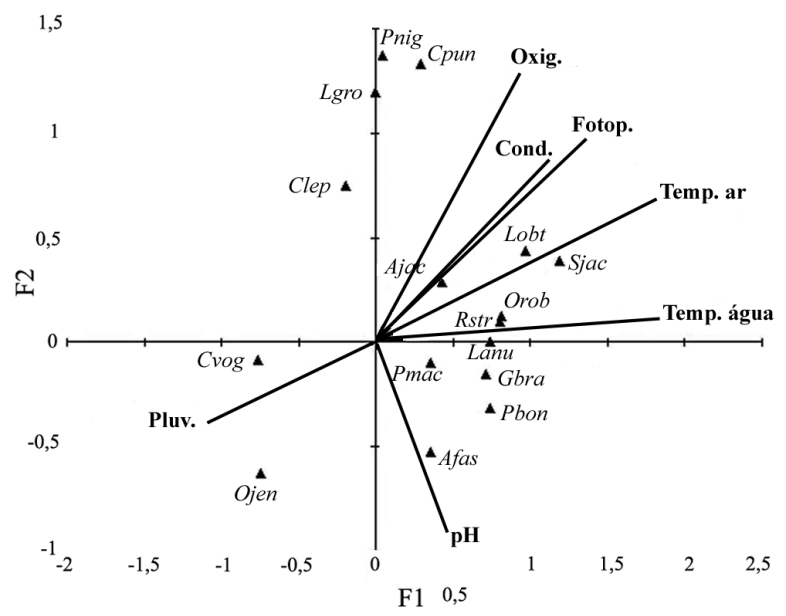

Figura 9. Gráficos de dispersão obtidos através da análise de correspondência canônica (ACC), para taxocenose de peixes da praia das Pombas, Parque Estadual de Itapuã, Viamão, RS (Cond., condutividade; Pluv., pluviosidade; Fotop., fotoperíodo; Oxig., oxigênio; Temp. água, temperatura da água; Temp. ar, temperatura do ar; Afas, Astyanax fasciatus; Ajac, Astyanax jacuhiensis; Calb, Cyanocharax alburnus; Clep, Crenicichla lepidota; Cpun, Crenicichla punctata; Cvog, Cyphocharax voga; Gbra, Geophagus brasiliensis; Hoan, Homodiaetus anisitsi; Lanu, Loricariichthys anus; Lgro, Lycengraulis grossidens; Lobt, Leporinus obtusidens; Odont(jov), Odontesthes jovens; Ojen, Oligosarcus jenynsii; Orob, Oligosarcus robustus; Pbon, Pachyurus bonariensis; Pmac, Pimelodus maculatus; Pnig, Parapimelodus nigribarbis; Rcad, Rineloricaria cadeae; Rstr, Rineloricaria strigilata; Sjac, Schizodon jacuiensis).
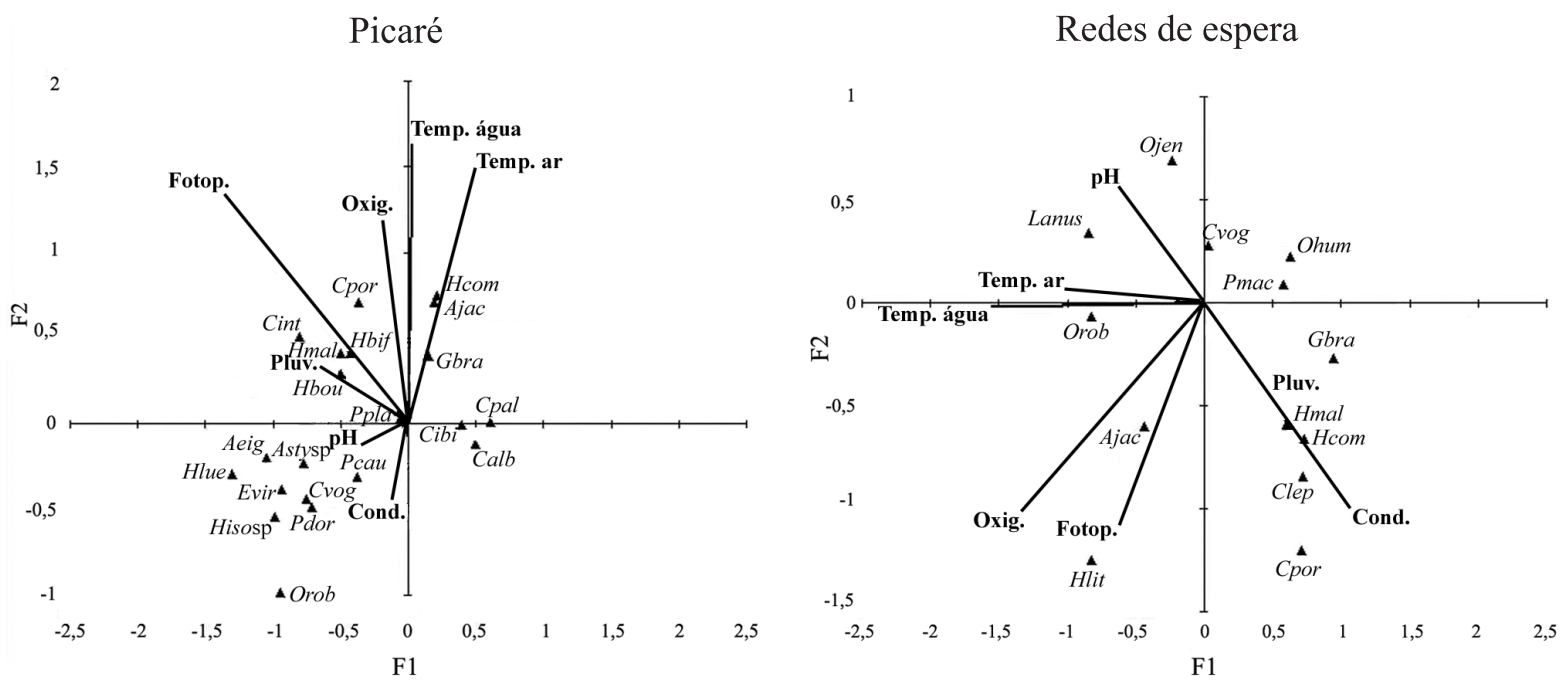

Figura 10. Gráficos de dispersão obtidos através da análise de correspondência canônica (ACC), para taxocenose de peixes da lagoa Negra, Parque Estadual de Itapuã, Viamão, RS (Cond., condutividade; Pluv., pluviosidade; Fotop., fotoperíodo; Oxig., oxigênio; Temp. água, temperatura da água; Temp. ar, temperatura do ar; Aeig, Astyanax eigenmanniorum; Ajac, Astyanax jacuhiensis; Astysp, Astyanax sp.; Calb, Cyanocharax alburnus; Cibi, Cheirodon ibicuhiensis; Cint, Cheirodon interruptus; Clep, Crenicichla lepidota; Cpal, Corydoras paleatus; Cpor, Cichlasoma portalegrense; Cvog, Cyphocharax voga; Evir, Eigenmannia virescens; Gbra, Geophagus brasiliensis; Hbif, Hyphessobrycon bifasciatus; Hbou, Hyphessobrycon boulengeri; Hcom, Hypostomus commersoni; Hisosp, Hisonotus sp.; Hlit, Hoplosternum littorale; Hlue, Hyphessobrycon luetkenii; Hmal, Hoplias malabaricus; Lanu, Loricariichthys anus; Ohum, Odontesthes humensis; Ojen, Oligosarcus jenynsii; Orob, Oligosarcus robustus; Pcau, Phalloceros caudimaculatus; Pdor, Pseudocorynopoma doriae; Pmac, Pimelodus maculatus; Ppla, Platanichthys platana).

( $77,31 \%$ para o picaré e $66,82 \%$ nas redes de espera). O fotoperíodo e a temperatura foram os fatores de maior importância nos dois ambientes. No entanto, a condutividade, o pH e o oxigênio dissolvido também foram importantes na distribuição das espécies na lagoa Negra.

\section{DISCUSSÃO}

De acordo com ReIs et al. (2003), para a ictiofauna de água doce da região Neotropical, a ordem Siluriformes é o grupo com o maior número de espécies, representando cerca de $38 \%$ de número total de espécies válidas, seguida 
Tabela II. Valores da análise de variância (ANOVA), para as variáveis ambientais mais explicativas na praia das Pombas e lagoa Negra, Parque Estadual de Itapuã, Viamão, RS, entre junho de 2002 e julho de 2003.

\begin{tabular}{|c|c|c|c|c|}
\hline & \multicolumn{4}{|c|}{ Praia das Pombas } \\
\hline & \multicolumn{2}{|c|}{ Picaré } & \multicolumn{2}{|c|}{ Redes de espera } \\
\hline \multirow{2}{*}{\multicolumn{5}{|c|}{$\frac{\text { Biomassa }}{\mathrm{t}=4,365-\operatorname{Pr}=0,001}$}} \\
\hline & & & & \\
\hline \multirow{3}{*}{\multicolumn{5}{|c|}{$\begin{array}{l}\text { Temp. do ar } \\
\text { pH } \\
\text { Condutividade }\end{array}$}} \\
\hline & & & & \\
\hline & & & & \\
\hline Oxigênio dissolvido & & & $\mathrm{t}=1,919-\operatorname{Pr}=0,084$ & $\mathrm{t}=1,736-\operatorname{Pr}=0,113$ \\
\hline \multicolumn{5}{|l|}{ Pluviosidade } \\
\hline \multirow[t]{4}{*}{ Fotoperíodo } & $\mathrm{t}=0,749-\operatorname{Pr}=0,471$ & $\mathrm{t}=-2,219-\operatorname{Pr}=0,005$ & & \\
\hline & \multicolumn{4}{|c|}{ Lagoa Negra } \\
\hline & \multicolumn{2}{|c|}{ Picaré } & \multicolumn{2}{|c|}{ Redes de espera } \\
\hline & Abundância & Biomassa & Abundância & Biomassa \\
\hline \multicolumn{5}{|l|}{ Temp. da água } \\
\hline \multicolumn{5}{|l|}{ Temp. do ar } \\
\hline $\mathrm{pH}$ & & $\mathrm{t}=-2,607-\operatorname{Pr}<0,05$ & & $\mathrm{t}=1,197-\operatorname{Pr}=0,259$ \\
\hline Condutividade & & & $\mathrm{t}=-2,210-\operatorname{Pr}=0,052$ & \\
\hline Oxigênio dissolvido & & & $\mathrm{t}=1,439-\operatorname{Pr}=0,181$ & $\mathrm{t}=0,652-\operatorname{Pr}=0,529$ \\
\hline Pluviosidade & $\mathrm{t}=-5,177-\operatorname{Pr}<0,05$ & & & \\
\hline Fotoperíodo & $\mathrm{t}=7,331-\operatorname{Pr}<0,05$ & $\mathrm{t}=2,708-\operatorname{Pr}<0,05$ & & \\
\hline
\end{tabular}

Tabela III. Resultados da análise de correspondência canônica (ACC) para a taxocenose da praia das Pombas e lagoa Negra, entre junho de 2002 e julho de 2003.

\begin{tabular}{|c|c|c|c|c|c|c|c|c|}
\hline \multirow{4}{*}{$\begin{array}{l}\text { Inércia Total } \\
\text { Variáveis Ambientais }\end{array}$} & \multicolumn{4}{|c|}{ Praia das Pombas } & \multicolumn{4}{|c|}{ Lagoa Negra } \\
\hline & \multicolumn{2}{|c|}{ Picaré } & \multicolumn{2}{|c|}{ Redes de espera } & \multicolumn{2}{|c|}{ Picaré } & \multicolumn{2}{|c|}{ Redes de espera } \\
\hline & \multicolumn{2}{|c|}{1,714} & \multicolumn{2}{|c|}{1,184} & \multicolumn{2}{|c|}{0,728} & \multicolumn{2}{|c|}{1,155} \\
\hline & $\mathrm{F} 1$ & $\mathrm{~F} 2$ & $\mathrm{~F} 1$ & $\mathrm{~F} 2$ & F1 & $\mathrm{F} 2$ & $\mathrm{~F} 1$ & $\mathrm{~F} 2$ \\
\hline Temp. da água & 0,868 & $-0,181$ & 0,908 & 0,065 & 0,017 & 0,815 & $-0,779$ & $-0,009$ \\
\hline Temp. do ar & 0,223 & $-0,032$ & 0,898 & 0,331 & 0,244 & 0,756 & $-0,489$ & 0,032 \\
\hline $\mathrm{pH}$ & 0,458 & $-0,725$ & 0,229 & $-0,470$ & $-0,182$ & $-0,061$ & $-0,306$ & 0,280 \\
\hline Condutividade & 0,307 & 0,059 & 0,575 & 0,441 & $-0,056$ & $-0,217$ & 0,528 & $-0,509$ \\
\hline Oxigênio dissolvido & 0,347 & 0,211 & 0,430 & 0,666 & $-0,092$ & 0,590 & $-0,687$ & $-0,511$ \\
\hline Pluviosidade & $-0,211$ & 0,176 & $-0,567$ & $-0,184$ & $-0,318$ & 0,186 & 0,229 & $-0,223$ \\
\hline Fotoperíodo & 0,730 & 0,129 & 0,677 & 0,483 & $-0,711$ & 0,672 & $-0,450$ & $-0,521$ \\
\hline$\%$ acumulada & 42,57 & 25,46 & 46,70 & 26,33 & 63,43 & 13,88 & 32,96 & 30,86 \\
\hline
\end{tabular}

pelos Characiformes, que representam cerca de 33\% das espécies. Lowe-McConNell (1987) destaca o predomínio desses dois grupos na ictiofauna sul-americana, especialmente em águas correntes. No presente estudo, estes dois grupos de peixes também foram predominantes.

A riqueza de espécies é um dos parâmetros mais difíceis de se determinar na região Neotropical, principalmente em virtude da grande diversidade de formas e hábitos apresentados pelos peixes (SANTOS \& FERREIRA, 1999). No presente estudo, foram identificadas 44 espécies de peixes na praia das Pombas e 44 na lagoa Negra. LuCENA et al. (1994), estudando a ocorrência e distribuição da ictiofauna da praia de Itapuã, também no lago Guaíba, encontraram 36 espécies de peixes. No entanto, estes autores utilizaram como amostrador somente o arrasto de margem (picaré), com esforço de seis arrastos/mês. GrossER \& HAHN (1981) compilaram uma lista com 46 espécies para esta mesma lagoa, porém, utilizando diferentes artes de pesca e explorando vários pontos deste ambiente. Algumas das espécies coletadas no presente estudo não haviam sido ainda registradas para esses ambientes (Tab. I).

A praia das Pombas caracteriza-se, principalmente por ser um ambiente de águas livres (RIO GRANDE DO SuL, 1997). Este fator reflete consideravelmente na ictiofauna local, sendo algumas espécies de peixes características deste tipo de ambiente, como Astyanax fasciatus, A. jacuhiensis e Cyanocharax alburnus, Cyphocharax voga, Pachyurus bonariensis e Rineloricaria strigilata, que foram as espécies mais abundantes e frequentes. A maior captura de espécies de peixes-rei (Odontesthes spp.) ocorreu neste ambiente e, segundo KLEEREKOPER (1945) e BECKER et al. (2003), estes peixes tendem a se concentrar em praias de fundo arenoso junto às manchas de juncos, características observadas apenas na praia das Pombas.

Uma espécie que merece destaque é Pachyurus bonariensis, que esteve entre as mais representativas tanto em número de indivíduos quanto em biomassa, nas capturas da praia das Pombas, uma vez que não foi capturada em coletas anteriores realizadas no Parque Estadual de Itapuã (Rio Grande do Sul, 1997). A distribuição original desta espécie compreende corpos d'água do Sistema do rio Uruguai, sendo considerada alóctone ao Sistema da laguna dos Patos (DufECH \& FIALHO, 2007). 
A alta abundância, e consequente biomassa, de Cyphocharax voga, obtidas no mês de junho de 2002 na praia das Pombas pode estar relacionado à alta pluviosidade neste mês. Os resultados da ANOVA e ACC das redes de espera confirmam esta hipótese. GALACATOS et al. (2004) relatam uma maior abundância de curimatídeos nas estações onde os valores de pluviosidade estavam mais elevados, em contraponto à estação de seca, onde houve ausência completa destes indivíduos.

A ictiofauna da lagoa Negra é composta basicamente por peixes de pequeno porte, como Astyanax eigenmanniorum, Cheirodon ibicuhiensis, $C$. interruptus, Cyanocharax alburnus, Hyphessobrycon bifasciatus, $H$. luetkenii e Hisonotus nigricauda, que ocorrem preferencialmente junto às margens, onde se concentra um grande número de macrófitas aquáticas. Resultados semelhantes foram obtidos por CASATTI et al. (2003), que encontraram uma predominância de pequenos caracídeos em locais com maior quantidade de macrófitas aquáticas, e por AgostinHo \& JÚLIo JR. (1999), estudando os peixes da bacia do alto rio Paraná, que relatam que as comunidades das lagoas marginais são compostas, em sua maioria, por espécies de pequeno porte e indivíduos jovens das espécies maiores.

A baixa similaridade ictiofaunística entre a praia das Pombas e a lagoa Negra e diferenças na abundância de espécies podem estar relacionados aos hábitats propícios para a alimentação e/ou desova destas espécies. Os dois ambientes estudados apresentaram características peculiares, que refletem sobre a ictiofauna de cada um destes locais. A vegetação marginal encontrada na praia das Pombas, representada principalmente pelos sarandis, caracteriza-se como um importante habitat para muitas espécies de peixes. Exemplares da família Cichlidae (Geophagus brasiliensis, Gymnogeophagus spp. e Crenicichla spp.) e Hoplias malabaricus foram capturados preferencialmente junto à vegetação, onde encontram abrigo para desova e para alimentação (HECK \& Crowder, 1991; WeAver et al., 1996). A importância da vegetação para peixes de água doce fornecendo refúgio contra predadores, áreas para forrageamento e locais para desova é bem documentada nos trabalhos de GORMAN \& Karr (1978), ArgermeIER \& Karr (1983), RozAS \& Odum (1987), Pusey et al. (1993) e Meschiatti et al. (2000).

A vegetação flutuante, principalmente as macrófitas, muito abundantes na lagoa Negra, consiste em um biótopo que apresenta uma alta produção e tem grande importância ecológica por servir de abrigo, fonte de alimento e local de desova para várias espécies de animais aquáticos e terrestres, como peixes e insetos (JunK, 1973; Welcomme, 1979; CAStro \& Arcifa, 1987). Os gimnotiformes encontrados na lagoa Negra são exemplos de organismos que dependem diretamente deste tipo de vegetação para sua sobrevivência. Eigenmannia trilineata, por exemplo, é encontrada em cardumes sob estas plantas, as quais são comprovadamente utilizadas na dieta da espécie, assim como os invertebrados que se associam às suas raízes (GIORA et al., 2005).

As espécies constantes neste estudo foram também as mais abundantes nos dois ambientes. Resultado similar foi encontrado por LuCENA et al. (1994) na praia de Itapuã, no lago Guaíba, RS, Smith \& Barrella (2000) no rio Sorocaba, SP, e Miranda \& Mazzoni (2003) no rio Tocantins, GO. GARUTTI (1988) afirma que a constância de ocorrência de espécies de peixes é importante na caracterização de qualquer ponto em um curso d'água. É também uma medida qualitativa que pode evidenciar as espécies migrantes ou residentes de uma comunidade, assim como o possível efeito das variações sazonais sobre as comunidades (UIEDA, 1984).

Segundo Lowe-McConnell (1975), em rios e sistemas interligados, a composição de espécies é mais dinâmica e, portanto, não haveria uma grande quantidade de espécies constantes. Resultados similares foram encontrados na praia das Pombas, a qual faz parte do lago Guaíba e possui ligação contínua com outros grandes corpos d'água (laguna dos Patos e rio Jacuí). A lagoa Negra, ao contrário, apresentou quantidade elevada de espécies constantes por ser um ambiente fechado (com conexão temporária com a laguna dos Patos) e, assim, não ocorre grande deslocamento das espécies de peixes de um ambiente a outro.

Através da análise da variação temporal dos resultados obtidos, pôde se verificar que houve variação na abundância, biomassa e na diversidade ao longo do ano, com valores mais elevados durante os meses quentes no hemisfério sul. Houve dependência destes atributos, principalmente, com a temperatura e com o fotoperíodo. Resultados semelhantes também foram observados por LuCENA et al. (1994), na praia de Itapuã. GalaCATos et al. (2004) constataram que a abundância, a riqueza de espécies e a diversidade variaram sazonalmente em todos os locais estudados. Segundo DAY et al. (1981) e MoyLE et al. (1986) este padrão de distribuição temporal é comumente evidenciado em ambientes de clima temperado e é influenciado pelas migrações sazonais para desova, ciclos reprodutivos e recrutamento de jovens (BоTTON \& Jones, 1990). As demais variáveis também foram importantes neste estudo, porém não tão evidentes.

GALACATOS et al. (2004), ao estudarem a ictiofauna de três ambientes distintos (rio, lagoa e riacho), encontraram padrão sazonal para as variações na temperatura, oxigênio dissolvido, $\mathrm{pH}$, condutividade e turbidez. Estes autores verificaram que as águas escuras da lagoa estudada apresentaram altas temperaturas, baixo $\mathrm{pH}$ e baixa condutividade comparados ao rio e ao riacho. De forma semelhante, houve relação entre alguns atributos da taxocenose dos ambientes estudados com o $\mathrm{pH}$, a condutividade e o oxigênio dissolvido. SúAREZ \& Petrere Jr. (2006), estudando as comunidades de peixes da bacia do rio Iguatemi, MS, constataram que a riqueza de espécies e a abundância de indivíduos estavam significativamente correlacionadas com o $\mathrm{pH}$, temperatura, oxigênio dissolvido e condutividade.

De acordo com Hook (1991), variações sazonais na abundância relativa são influenciadas pelo período do ano onde ocorre a desova ou o recrutamento. Cada espécie alcança o máximo da abundância numérica quando os juvenis são recrutados para a população. A sazonalidade reprodutiva em peixes de regiões temperadas estaria associada a um aumento do fotoperíodo e da disponibilidade de alimento, além da temperatura (McKaye, 1984; PAYne, 1986). No entanto, em ambientes 
tropicais, variações no fotoperíodo e na temperatura são muito pequenas, sendo a pluviosidade e a disponibilidade de habitats os fatores responsáveis pela sazonalidade em rios, riachos e lagos (Goulding, 1980). No Rio Grande do Sul o clima é temperado, entretanto, os períodos de alta ou baixa pluviosidade não são bem definidos. Estudos nesta região (AzEvEDo et al., 2000; KaLIL et al., 2005) indicam que o período reprodutivo das espécies está normalmente associado ao fotoperíodo e à temperatura.

Os resultados obtidos neste estudo permitem concluir que a taxocenose de peixes dos dois ambientes estudados, mesmo fazendo parte de um mesmo Sistema Hidrográfico, apresenta diferenças na composição de espécies. Isto se deve às diferenças nas características ambientais de cada local, uma vez que um dos ambientes se constitui em uma praia e o outro em uma lagoa. A abundância, a biomassa e a diversidade, variaram ao longo do ano, mostrando seu caráter sazonal. Além disso, estes fatores tiveram relação com as variáveis ambientais, sendo a temperatura e o fotoperíodo os mais importantes para a determinação da distribuição de espécies na praia das Pombas e na lagoa Negra.

Agradecimentos. Ao Prof. Dr. Luiz R. Malabarba pelas sugestões no decorrer do trabalho e aos colegas do Laboratório de Ictiologia (UFRGS) pelo auxílio nas saídas de campo. Ao CNPq (proc. 464545/00-5 e 476821/2003-7) e à CAPES pela bolsa concedida.

\section{REFERÊNCIAS BIBLIOGRÁFICAS}

Abes, S. S. \& Agostinho, A. A. 2001. Spatial patterns in fish distribuitions and structure of the ichthyocenosis in the Água de Nanci stream, upper Paraná river basin, Brazil. Hydrobiologia 445:217-227.

Agostinho, A. A. \& Júlio JR., H. F. 1999. Peixes da bacia do alto rio Paraná. In: Lowe-McConnell, R. H. ed. Estudos ecológicos de comunidades de peixes tropicais. São Paulo, Editora da Universidade de São Paulo. p.374-400.

Angermeier, P. L. \& KarR, J. R. 1983. Fish communities along environmental gradients in a system of tropical streams. Environmental Biology of Fishes 9(2):117-135.

Arrington, D. A. \& Winemiller, K. O. 2006. Habitat affinity, the seasonal flood pulse, and community assembly in the litoral zone of a Neotropical floorplain river. Journal of the North American Benthological Society 25:126-141.

Azevedo, M. A.; Malabarba, L. R. \& Fialho, C. B. 2000. Reproductive biology of the inseminated Glandulocaudinae Diapoma speculiferum Cope (Actinopterygii: Characidae). Copeia 2000(4):983-989.

Becker, F. G.; Bruschi Jr., W. \& Peret, A. C. 2003. Age and growth of three Odontesthes species from Southern Brazil (Atherinopsidae), with reference to phylogenetic constraints in their life-history. Brazilian Journal of Biology 63(4):567-578

Botton, D. L. \& Jones, K. K. 1990. Species composition, distribution and invertebrate prey of fish assemblages in the Columbia River Estuary. Progress in Oceanography 25:243-270.

CAIn, S. A. 1938. The species-area curve. American Midland Naturalist 19:573-581.

Casatti, L.; Mendes, H. F. \& Ferreira, K. M. 2003. Aquaticmacrophytes as feeding site for small fishes in the Rosana Reservoir, Paranapanema River, Southeastern Brazil. Brazilian Journal of Biology 63(2):213-222.

Castro, R. M. C. \& Arcifa, M. S. 1987. Comunidades de peixes de reservatórios no sul do Brasil. Revista Brasileira de Biologia 47(4):493-500.

Colwell, R. K. 2000. EstimateS 7.5: statistical estimation of species richness and shared species from samples.
Disponível em: <http://viceroy.eed.uconn.edu/estimates> Acesso em: 04.03.2005.

Dajoz, R. 1978. Ecologia Geral. Petrópolis, Vozes. 472p.

Day, J. H.; Blaber, S. J. M. \& Wallace, J. H. 1981. Estuarine fishes. In: DAY, J. H. ed. Estuarine ecology with particular reference to southern Africa. Rotterdam, A. A. Balkema. p.199-221.

Dufech, A. P. S. \& Fialho, C. B. 2007. Biologia populacional de Pachyurus bonariensis Steindachner, 1879 (Perciformes, Sciaenidae), uma espécie alóctone no sistema hidrográfico da Laguna dos Patos, Brasil. Biota Neotropica 7(1):105-110.

Galacatos, K.; Barriga-Salazar, R. \& Stewart, D. J. 2004. Seasonal and habitat influences on fish communities within the lower Yasuni River basin of the Ecuadorian Amazon. Environmental Biology of Fishes 71:33-51.

Galacatos, K.; Stewart, D. J. \& Ibarra, M. 1996. Fishy community patterns of lagoons and associated tributaries in the Ecuadorian Amazon. Copeia 1996:875-894.

Garutti, V. 1988. Distribuição longitudinal da ictiofauna em um córrego da região noroeste do Estado de São Paulo, bacia do rio Paraná. Revista Brasileira de Biologia 48:747-759.

Giora, J.; Fialho, C. B. \& Dufech, A. P. 2005. Feeding habits of Eigenmannia trilineata López \& Castello, 1966 (Teleostei, Sternopygidae) of Parque Estadual de Itapuã, Rio Grande do Sul, Brazil. Neotropical Ichthyology 3(2):291-298.

Gorman, O. T. \& KarR, J. R. 1978. Habitat structure and stream fish communities. Ecology 59(3):507-515.

Goulding, M. 1980. The fishes and the forest: explorations in Amazon natural history. Berkeley, Univ. California. 280 p.

Grenoulllet, G.; Pont, D. \& Seip, K. L. 2002. Abundance and species richness as a function of food resources and vegetation structure: juvenile fish assemblages in rivers. Ecography 25:641-650

Grosser, K. M. \& Hahn, S. D. 1981. Ictiofauna da Lagoa Negra Parque Estadual de Itapuã, município de Viamão, Rio Grande do Sul, Brasil. Iheringia, Série Zoologia, 59:45-64.

Heck, K. \& Crowder, L. 1991. Habitat structure and predatorprey interactions in vegetated aquatic systems. In: BELL, S.; Coy, M. C. E. \& Mushinsky, H. eds. Habitat Structure: the Physical Arrangement of Objects in Space. New York, Chapman \& Hall. p.282-299.

Henderson, P. A. \& Crampton, W. G. R. 1997. A comparison of fish diversity and abundance between nutrient-rich and nutrientpoor lakes in the Upper Amazon. Journal of Tropical Ecology 13:175-198.

Henderson, P. A. \& Hamilton, H. F. 1995. Standing crop and distribution of fish in drifting and attached floating meadow within an Upper Amazonian varzea lake. Journal of Fish Biology 47:266-276

Hook, J. H. 1991. Seasonal variation in relative abundance and species diversity of fishes in south bay. Contributions in Marine Science 32:127-141.

JACKSON, D. A. \& HARveY, H. H. 1989. Biogeographic associations in fish assemblages: Local vs. Regional processes. Ecology 70:1472-1484

Jackson, D. A.; Peres-Neto, P. R. \& Olden, J. D. 2001. What controls who is where in freshwater fish communities - the roles os biotics, abiotics, and spatial factors. Canadian Journal of Fisheries and Aquatics Sciences 58:157 170

JunK, W. J. 1973. Investigations on the ecology and production biology of the floating meadows (Paspalo-Echinochloetum) on the Middle Amazon. Part 2: The aquatic fauna in the roo zone of the floating vegetation. Amazoniana 4:9-12.

Kalil, T. G.; Azevedo, M. A.; Malabarba, L. R. \& Fialho, C. B. 2005. Reproductive biology and development of sexually dimorphic structures in Aphyocharax anisitsi (Ostariophysi: Characidae). Neotropical Ichthyology 3(3):433-438.

KleErekoper, H. 1945. O peixe-rei. Rio de Janeiro, Ministério da Agricultura, Serviço de Informação Agrícola. 98p.

KöPPEN, W. 1948. Climatologia; con un estudio de los climas de la tierra. México, Fondo de Cultura Economica. 478p.

Layman, C. A. \& Winemiller, K. O. 2005. Patterns of habitat segregation among large fishes in a Venezuelan floodplain river. Neotropical Ichthyology 3(1):111-117. 
Lowe-McConnell, R. H. 1975. Fish communities in tropical freshwaters. New York, Longman Inc. 337p.

1987. Ecological studies in tropical fish communities. Cambridge, Cambridge University. 382p.

Lucena, C. A. S.; Jardim, A. S. \& Vidal, E. S. 1994. Ocorrência, distribuição e abundância da fauna de peixes da praia de Itapuã, Viamão, Rio Grande do Sul, Brasil. Comunicações do Museu de Ciências e Tecnologia - PUCRS, Série Zoologia, 7:3-27.

Martin-Smith, K. M. 1998. Relationships between fishes and habitat in rainforest streams in Sabah, Malaysia. Journal of Fish Biology 52:458-482.

Matthews, W. L. \& Robinson, H. W. 1998. Influence of drainage connectivity, drainage area and regional species richness of fishes of the interior highlands in Arkansas. The American Midland Naturalist 139(1):1-19.

Mazzoni, R. \& Lobón-Cerviá, J. 2000. Longitudinal structure, density and production rates of a neotropical stream fish assemblage: the river Ubatiba in the Serra do Mar, Southeast Brazil. Ecography 23:588-602.

MCKAYE, K. R. 1984. Behavioral aspects of cichlids reproductive strategies: patterns of territoriality and brood defense in Central American substratum spawner and African mouth brooders. In: Pоттs, G. W. \& Wooton, R. J. eds. Fish reproduction: strategies and tatics. London, Academic. p. 245-273.

Menegat, R.; Porto, M. L.; Carraro, C. C. \& Fernandes, L. A. D. 1998. Atlas Ambiental de Porto Alegre. Porto Alegre, Editora da Universidade/UFRGS. 228p.

Meschiatti, A. J.; Arcifa, M. S. \& Fenerich-Verani, N. 2000. Fish communities associated with macrophytes in Brazilian floodplain lakes. Environmental Biology of Fishes 58:133-143.

Miranda, J. C. \& Mazzoni, R. 2003. Composição da ictiofauna de três riachos do alto rio Tocantins - GO. Biota Neotropica 3(1): $1-12$

Moyle, P. B.; Daniels, R. A.; Herbold, B. H. \& Baltz, D. M. 1986. Patterns of distribuition and abundance of a non-coevolved assemblage of estuarine fishes in California. Fishery Bulletin 84:105-117.

OberdorfF, T.; Hugheny, B. \& Vigneron, T. 2001. Is assemblage variability related to environmental variability? An answer for riverine fish. Oikos 93:419-428.

PAYNe, A. I. 1986. The ecology of tropical lakes and rivers. New York, John Wiley. 310p

Pérez-López, F. J \& Sola-Fernández, F. M. 1993. Divers: Programa para el cálculo de los índices de diversidade. Disponível em: <http://perso.wanadoo.es/ip-1/descargas $>$. Acesso em 29.06.2004.

Pielou, E. C. 1975. Ecological diversity. New York, John Wiley \& Sons. 165p.

Power, M. E. 1983. Grazing ecology of tropical freshwater fishes to different scales of variation in their food. Environmental Biology of Fishes 9:103-115.

Pusey, B. J.; Arthington, A. H. \& Read, M. G. 1993. Spatial and temporal variation in fish assemblage structure in the Mary River, southeastern Queensland: the influence of habitat structure. Environmental Biology of Fishes 37:355-380.

Reis, R. E.; Kullander, S. O. \& Ferraris Jr., C. J. 2003. Check list of the freshwater fishes of South and Central
America. Porto Alegre, EDIPUCRS. 742p.

Rio Grande do Sul. 1997. Plano de Manejo do Parque Estadual de Itapuã - RS. Porto Alegre, Departamento de Recursos Naturais Renováveis. 158p.

RodrígueZ, M. A. \& LewIS, W. M. 1994. Regulation and stability in fish assemblages of neotropical floorplain lakes. Oecologia 99:166-180.

1997. Structure of fish assemblages along environmental gradients in floodplain lakes of the Orinoco River. Ecological Monographs 67:109-128

Rozas, L. P. \& Odum, W. E. 1987. Use of tidal freshwater marshes by fishes and macrofaunal crustaceans along a marsh streamorder gradient. Estuarine 10(1):36-43

Santos, G. M. \& Ferreira, E. J. G. 1999. Peixes da Bacia Amazônica In: Lowe-McConnell, R. H. ed. Estudos ecológicos de comunidades de peixes tropicais. São Paulo, Editora da Universidade de São Paulo. p.345-373.

Smith, W. S. \& Barrella, W. 2000. The ichthyofauna of the marginal lagoons of the Sorocaba River, SP, Brazil: Composition, abundance and effect of the anthropogenic actions. Revista Brasileira de Biologia 60(4):627-632.

Súarez, Y. R. \& Petrere JR., M. 2006. Gradientes de diversidade nas comunidades de peixes da bacia do rio Iguatemi, Mato Grosso do Sul, Brasil. Iheringia, Série Zoologia, 96(2):197-204

Tejerina-Garro, F. L.; Fortin, R. \& Rodríguez, M. A. 1998. Fish community structure in relation to environmental variation in floodplain lakes of the Araguaia River, Amazon Basin. Environmental Biology of Fishes 51:399-410.

Ter Braak, C. J. F. 1986. Canonical Correspondence Analysis: A new eigenvector technique for multivariate direct gradient analysis. Ecology 67(5):1167-1179.

Tonn, W. M.; Magnuson, J. J.; Rask, M. \& Toivonen, J. 1990. Intercontinental comparison of small-lake fish assemblages: The balance between local and regional processes. Americam Naturalist 136:345-375.

UIEDA, V. S. 1984. Ocorrência e distribuição dos peixes em um riacho de água doce. Revista Brasileira de Biologia 44(2):203-213.

UiedA, V. S. \& BARReto, M. G. 1999. Composição da ictiofauna de quatro trechos de diferentes ordens do rio Capivara, Bacia do Tietê, Botucatu, São Paulo. Revista Brasileira de Zoociências 1:55-67.

VolKMER-Ribeiro, C. 1981. Limnologia e a vegetação de macrófitas na lagoa Negra, Parque Estadual de Itapuã, Rio Grande do Sul Iheringia, Série Botânica, 27:38-59.

Vono, V. \& Barbosa, F. A. R. 2001. Habitats and littoral zone fish community structure of two natural lakes in southeast Brazil. Environmental Biology of Fishes 61:371-379.

Weaver, M. J.; Magnuson, J. J. \& Clayton, M. K. 1996. Habitat heterogeneity and fish community structure: Inference from north temperate lakes. American Fisheries Society Symposium 16:335-346.

Welcomme, R. L. 1979. Fisheries ecology of floodplain rivers. New York, Longmann. 317p.

Wootton, R. J. 1990. Ecology of teleost fishes. London, Chapman and Hall. 404p.

ZAR, J. H. 1999. Biostatistical Analysis. 4 th ed. New Jersey, Prentice-Hall. 663p.

Recebido em abril de 2008. Aceito em novembro de 2008. ISSN 0073-4721

Artigo disponível em: www.scielo.br/isz 